\title{
THE SIGN OF PLASTIC POWER IN THE GRAPHICAL TREATMENT OF PROBLEMS OF PLANE PLASTIC FLOW*
}

\author{
BY WILLIAM PRAGER (Brown University)
}

1. Introduction. A graphical method of treating problems of plane plastic flow has been described in an earlier report, paper [1]**. This method uses the geometrical relations between the plane of flow (physical plane), the stress plane, and the hodograph plane. The coordinates of a point in the stress plane give the stress components $\sigma_{x}$ and $\tau_{x y}$ at the corresponding point in the physical plane. The slip lines in the physical plane are mapped on to the cycloids in the stress plane that are described by the points of the Mohr circles as these roll without sliding on the lines $\tau_{x y}= \pm k$. The radius vector of a point in the hodograph plane gives the velocity at the corresponding point in the physical plane. A line element of a slip line in the physical plane is orthogonal to its images in the stress and hodograph planes. These relations are used to construct, mesh by mesh, the slip line net in the physical plane and its images in the stress and hodograph planes. Before, however, a slip line field obtained in this manner can be accepted as the solution to the considered problem, it must be shown that the plastic power is non-negative throughout this slip line field.

A method of investigating the sign of the plastic power for a tentative solution has been described by Green [2]. Since Green's criterion involves the difference of two expressions that depend on the radii of curvature of the slip lines, it is not always easy to apply. An alternative criterion, which is believed to be more convenient than Green's is given in this report.

2. Shear stresses acting on a mesh of the slip line net. Let the rectangle in Fig. 1a

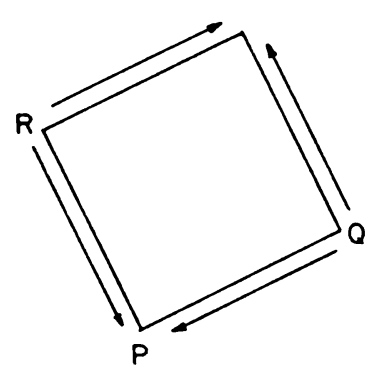

(0)

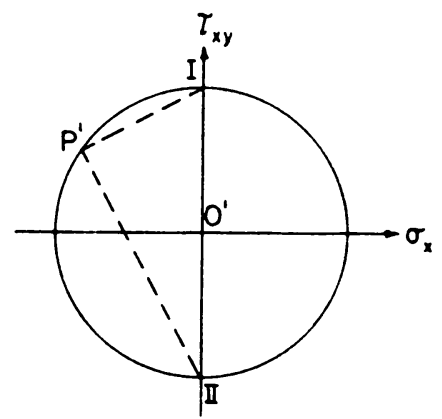

(b)

Fig. 1

represent a small mesh of the slip line net. It will always be possible to choose the corner $P$ of this mesh so that the direction of the side $P Q$ that falls on the first slip line through $P$ and the direction of the other side $P R$ through $P$ form a right-handed system in the plane of flow. It will be assumed in the following that the point $P$ has been chosen in this manner.

*Received March 25, 1955. The results presented in this paper were obtained in the course of research sponsored by Watertown Arsenal Laboratories, under Contract DA-19-020-ORD-2598.

${ }^{* *}$ Numbers in square brackets refer to the Bibliography at the end of the report. 
To determine the directions of the shear stresses transmitted on to the material inside the considered mesh, we use the Mohr circle of Fig. 1b. Since the superposition of a hydrostatic state of stress does not change the direction of the shearing stresses, we do not restrict the generality of our discussion by assuming the origin $O^{\prime}$ of the stress plane to coincide with the center of this circle. If the highest and lowest points of this circle are denoted by $I$ and $I I$, respectively, and if $P^{\prime}$ is the pole of this circle, the lines $P^{\prime} I$ and $P^{\prime} I I$ are parallel to the shear lines $P Q$ and $P R$, respectively. To find the direction of the shear stress transmitted across the side $P Q$ on to the material inside the mesh, we must rotate the exterior normal to this side by a right angle in the same sense in which $O^{\prime} I$ must be rotated by a right angle to coincide with the positive $\sigma_{x}$ axis. This furnishes the shear stresses indicated in Fig. 1a. For the plastic power to be positive, the shear rate at $P$ must therefore correspond to a decrease of the right angle at $P$.

3. Shear rate. As can be seen from Fig. 2, the rate at which the right angle at $P$

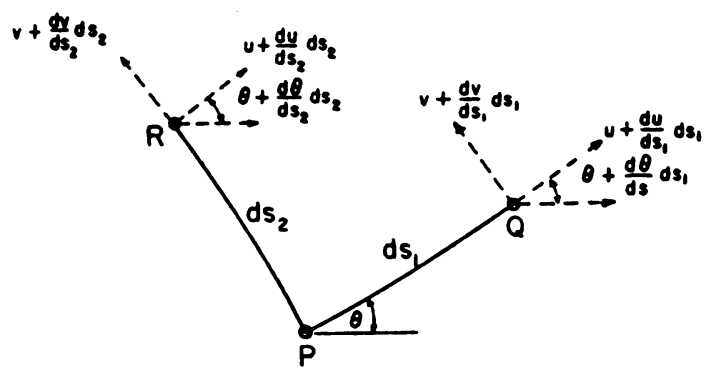

Fig. 2

is decreasing is given by

$$
\gamma=\frac{d v}{d s_{1}}+u \frac{d \theta}{d s_{1}}+\frac{d u}{d s_{2}}-v \frac{d \theta}{d s_{2}},
$$

where $u$ and $v$ are the components of the velocity vector in the first and second shear directions $(P Q$ and $P R)$, respectively, and $\theta$ is the angle between the first shear direction $(P Q)$ and a fixed direction.

As is well known from the theory of the slip line field, the velocity components $u$ and $v$ satisfy the following conditions along the slip lines:

$$
\frac{d u}{d s_{1}}-v \frac{d \theta}{d s_{1}}=0, \quad \frac{d v}{d s_{2}}+u \frac{d \theta}{d s_{2}}=0 .
$$

When the values of $d \theta / d s_{1}$ and $d \theta / d s_{2}$ obtained from (2) are substituted into (1), the following relation is obtained:

$$
\gamma=\frac{d v}{d s_{1}}+\frac{u}{v} \frac{d u}{d s_{1}}+\frac{d u}{d s_{2}}+\frac{v}{u} \frac{d v}{d s_{2}} .
$$

With $q^{2}=\frac{1}{2}\left(u^{2}+v^{2}\right)$, Eq. (3) can be written in the form

$$
\gamma=\frac{1}{u v}\left[u \frac{d q}{d s_{1}}+v \frac{d q}{d s_{2}}\right] .
$$


The bracket in Eq. (4) is positive, if the speed increases as one moves from $P$ in the direction of the resulting velocity to a neighboring point $P_{1}$ on the stream line through $P$. If the images of the points $P$ and $P_{1}$ in the hodograph plane are denoted by $P^{\prime \prime}$ and $P_{1}^{\prime \prime}$, the bracket in (4) is therefore positive, if $P^{\prime \prime}$ is closer to the origin $O^{\prime \prime}$ of the hodograph plane than $P_{1}^{\prime \prime}$. As the signs of $u$ and $v$ are readily determined, we have therefore a convenient criterion for the sign of the right-hand side of (4), that is for the sign of the plastic power. For a steady flow, in particular, this criterion requires that the particle that is instantaneously at $P$ should be accelerating or decelerating according to whether $u$ and $v$ have the same signs or not.

\title{
BibLiOGRAPHY
}

1. W. Prager, Trans. Roy. Inst. Technology, Stockholm, No. 65 (1953)

2. A. P. Green, J. Mech. Phys. Solids 2, 73, 296 (1954)

\section{NECESSARY AND SUFFICIENT CONDITIONS FOR THE EXISTENCE OF NEGATIVE SPECTRA*}

\author{
By C. R. PUTNAM (Purdue University)
}

Let $f(t)$ be a real-valued continuous function on the $t$-interval $0 \leqq t \leqq 2 T$ and let $x=x(t)$ be a real-valued solution $(\not \equiv 0)$ of the differential equation

$$
x^{\prime \prime}+f(t) x=0 \text {. }
$$

If $f^{+}=f$ or 0 according as $f \geqq 0$ or $f<0$, and if $x(a)=x(b)=0$, where $a<b$, then there holds the inequality

$$
\int_{a}^{b} f^{+}(t) d t>4 /(b-a)
$$

due essentially to Liapounoff (see [1]; also [8]). Moreover, according to [4] (see also [1]), the constant 4 of (2) is the best possible, in the sense that (2) need not hold (for arbitrary $f$ ) if the 4 is replaced by $4+\epsilon$, where $\epsilon>0$. Hence, it is easy to see that the inequality

$$
T \int_{0}^{T}\left[f^{+}(t)+f^{+}(2 T-t)\right] d t \quad\left(=T \int_{0}^{2 T} f^{+}(t) d t\right)>2
$$

is necessary, but $T \int_{0}^{2 T} f^{+}(t) d t>2+\epsilon$ is not, in order that the Sturm-Liouville boundary value problem

$$
L(x)+\lambda x=0 \quad\left[L(x) \equiv x^{\prime \prime}+f x\right], \quad x(0)=x(2 T)=0
$$

possess an eigenvalue $\lambda<0$ (or even $\leqq 0$ ).

It will be shown in this note that there is a sufficient criterion similar to (3). In fact,

*Received June 11, 1954. This work was supported by the National Science Foundation research grant NSF-G-481. 